\title{
Mortalidade em recém-nascidos de baixo peso ao nascer: limites e desafios para o acesso universal
}

\author{
Mario J. Setumba ${ }^{a}$ Manuel Simão ${ }^{a}$ Maria Conceição M. Silva ${ }^{a}$ \\ Carmen Gracinda S. Scochi ${ }^{b}$ Juliana C. Pina ${ }^{c}$ Debora F. Mello ${ }^{b}$ \\ aDepartamento de Enfermagem, Instituto Superior de Ciências da Saúde, Universidade Agostinho Neto, \\ Luanda, Angola; 'bepartamento Enfermagem Materno Infantil e Saúde Pública, Escola de Enfermagem de Ribeirão \\ Preto, Universidade de São Paulo, Ribeirão Preto, Brazil; ' Departamento de Enfermagem, Universidade Federal de \\ Santa Catarina, Florianópolis, Brazil
}

\section{Palavras-Chave}

Recém-nascido · Baixo peso ao nascer · Mortalidade neonatal $\cdot$ Acesso à saúde $\cdot$ Equidade em saúde

\section{Resumo}

Objetivo: analisar a mortalidade neonatal de recém-nascidos angolanos de baixo peso ao nascer, os limites e desafios para a promoção do acesso à saúde no contexto local. Método: Estudo descritivo, retrospectivo, com base em dados secundários de 2,100 bebês de baixo peso ao nascer, a partir de registros hospitalares de 2007 a 2013, em Kuito-Angola. Resultados: No período, a prevalência do baixo peso ao nascer foi de $7.5 \%$, com 437 óbitos e $70.3 \%$ de mortalidade neonatal. Foram encontradas as seguintes informações maternas e dos recém-nascidos: idade menor que 19 anos, doenças durante a gestação e gestantes que não participaram satisfatoriamente de consultas no pré-natal, extremo baixo peso ao nascer, muito baixo peso, índice de Apgar menor que 6 e idade gestacional menor que 37 semanas, as quais são consonantes com condições de vulnerabilidades. Esses fatores e as dimensões a eles interrelacionadas podem aumentar a ocorrência da mortalidade neonatal e denotar dificuldades ao acesso à saúde. Conclusão: Há expressivo número de mortes evitáveis, relacionadas aos grupos reduzíveis por: ações de imunoprevenção, atenção à mulher na gestação, parto e ao recém-nascido, diagnóstico e tratamento adequados, e promoção da saúde. Os critérios de evitabilidade de óbitos em crianças oferecemuma ampla análise das condições sociais e dos serviços de saúde, levando-se em conta uma adequada atenção ao pré-natal, ao parto, ao nascimento e no seguimento longitudinal, em busca do acesso à saúde com equidade, intervenções profissionais qualificadas e gestão do cuidado integral à saúde.

(c) 2018 The Author(s.) Published by S. Karger AG, Basel on behalf of NOVA National School of Public Health

\section{Mortality in Newborns with Low Birth Weight: Limits and Challenges for Universal Access}

\section{Keywords}

Newborn · Low birth weight · Neonatal mortality · Access in health · Equity in health

\section{KARGER}

E-Mail karger@karger.com www.karger.com/pjp

Karger Open access (c) 2018 The Author(s). Published by S. Karger AG, Basel on behalf of NOVA National School of Public Health

This article is licensed under the Creative Commons AttributionNonCommercial-NoDerivatives 4.0 International License (CC BYNC-ND) (http://www.karger.com/Services/OpenAccessLicense) Usage and distribution for commercial purposes as well as any distribution of modified material requires written permission.
Dr. Debora Falleiros de Mello, $\mathrm{PhD}$

Departamento Enfermagem Materno Infantil e Saúde Pública Escola de Enfermagem de Ribeirão Preto, Universidade de São Paulo Av. Bandeirantes, 3900, Ribeirão Preto, SP 14040190 (Brazil) E-Mail defmello@eerp.usp.br 


\begin{abstract}
Objective: To analyze neonatal mortality of newborns with low birth weight from Angola and the limits of and challenges for the promotion of universal access to health in local context. Method: This is a descriptive retrospective study based on secondary data of hospital records of 2,100 low-birth-weight infants in Angola from 2007 to 2013. Results: During this period, the prevalence of lowbirth-weight infants was $7.5 \%$, with 437 deaths, and $70.3 \%$ neonatal mortality. The following maternal and newborn characteristics were found: maternal age <19 years, diseases during pregnancy, and low adherence to prenatal consultations, extreme low birth weight, very low birth weight, Apgar score of $<6$, and gestational age $<37$ weeks; all of which are consistent with conditions of social vulnerability. These factors and their interrelations can increase the occurrence of neonatal mortality and denote difficulties to access health. Conclusion: There is a significant number of preventable death which could be reduced in these groups by: vaccination actions, care for women during pregnancy, childbirth and newborn care, diagnosis, treatment, and health promotion. Death in these children could be avoided by a broad analysis of the social conditions and health services, taking into account adequate prenatal care, delivery, birth, and longitudinal follow-up, and searching for ways to access health with fairness, with qualified professional interventions, and the management of integral health care.
\end{abstract}

(c) 2018 The Author(s). Published by S. Karger AG, Basel on behalf of NOVA National School of Public Health

\section{Introdução}

Muitos esforços mundiais têm sido empregados para melhorar as condições de saúde das crianças com o objetivo de reduzir a morbimortalidade na infância [1]. Para a OMS a saúde materna e infantil é a prioridade central do desenvolvimento humano, que requer esforços para a redução de mortes neonatais, empreendidos desde a virada do milênio, na perspectiva do acesso e cobertura universal em saúde [2].

Em âmbito internacional, a Declaração do Milênio, elaborada em 2000, considerou as seguintes prioridades: reduzir a mortalidade infantil, melhorar a saúde das gestantes, acabar com a fome e a miséria, educação básica para todos, igualdade entre sexos e valorização da mulher, combater a AIDS, a malária e outras doenças, qualidade de vida e respeito ao meio ambiente, e todos trabalhando pelo desenvolvimento das populações $[3,4]$. A era dos
Objetivos de Desenvolvimento do Milênio está sendo redimensionada e a agenda do setor saúde pós-2015 traz a visão da cobertura universal de saúde, com caráter de sistemas de saúde mais sólidos e equitativos, como um marco orientador do desenvolvimento global [2]. A cobertura universal da saúde está sendo vista como uma terceira transição sanitária global, em busca de oferecer a todas as pessoas os serviços de saúde de que necessitam, com acesso universal e igualitário aos cuidados de saúde $[5,6]$.

A mortalidade em crianças reflete as condições de vida de uma população, e configura um indicador que demonstra o estado de desenvolvimento de uma sociedade, extremamente influenciado por determinantes sociais, ambientais, econômicos e de saúde, sendo fundamental que os países conheçam seus indicadores e priorizem intervenções eficazes [1].

A estimativa para 2030, em termos mundiais, é de que a mortalidade no período neonatal será alta, proporcionalmente, mas o nível de aumento provavelmente tem variação por causa das complicações de partos pré-termo e as relacionadas ao período intrapartum, permanecendo como a principal causa de mortes neonatais e menores de 5 anos [1]. A menos que o progresso seja acelerado, a previsão é de que mais de 60 países não cumprirão os Objetivos de Desenvolvimento Sustentável de colocar fim às mortes evitáveis de recém-nascidos em 2030, e metade deles não atingirão a meta de 12 mortes por 1,000 nascidos vivos até $2050[7,8]$.

As crianças africanas têm uma proporção elevada de óbitos, quando comparadas com as crianças do restante do mundo. $\mathrm{Na}$ África, encontram-se 23 dos 24 países com mortalidade infantil acima de 100 por 1000 nascidos vivos, e constitui motivo de particular preocupação a lentidão com que a mortalidade neonatal e infantil está a reduzir [1]. As principais causas de morte dos recém-nascidos africanos estão relacionadas às infecções, prematuridade e asfixia perinatal $[1,7,8]$. Em Angola, a mortalidade na infância apresenta diferenças entre áreas urbanas (43 mortes em cada 1,000 nascidos vivos) e áreas rurais (61 mortes em cada 1,000 nascidos vivos), com expressivo contingente de óbitos antes de completar quatro semanas de vida [9].

Aspectos da mortalidade neonatal em bebês de baixo peso ao nascer em contexto de privação socioeconômica e diversidade cultural, com altos níveis de necessidades de saúde não atendidas [10] e iniquidades [11] são pouco difundidos no que se refere aos limites para acesso à saúde. Diante da importância do acesso universal aos cuidados de saúde $[2,5,6]$ e das iniquidades sociais para a saúde infantil entre os países [12], é relevante investigar aspectos da mortalidade neonatal e das circunstâncias de 
óbitos evitáveis nos referidos contextos, bem como os desafios para promover o acesso universal à saúde. Assim, esta investigação teve por objetivo analisar a mortalidade neonatal de recém-nascidos (RN) angolanos de baixo peso ao nascer (BPN) e os limites e desafios para a promoção do acesso à saúde no contexto local.

\section{Método}

Estudo descritivo e retrospectivo, de natureza quantitativa, com base em dados secundários, com obtenção de informações de saúde de RN de BPN e suas mães, de relevância para uma análise de situações de saúde [13].

Esta investigação foi desenvolvida na Maternidade do Hospital Geral do Bié (HGB), localizada no município de Kuito, na província do Bié-Angola. Apesar do crescimento econômico, os indicadores apontam que a maioria da população angolana continua a viver abaixo da linha da pobreza, com uma fecundidade média de 6,7 filhos por mulher e uma estimativa de menores de 15 anos de $48 \%$ da população geral [14]. Bié é uma região administrativa angolana que tem 09 municípios, 39 comunas, 1,924 aldeias e 619 embalas, e foi uma das regiões mais afetadas pela guerra que durou quase 30 anos, finalizada em 2002 [14].

A maternidade do HGB tem uma capacidade de 77 leitos, uma sala de parto, uma sala pós-parto, um bloco operatório, com uma média de 335 partos por mês, e uma unidade neonatal com capacidade de 09 berços e 03 incubadoras. Há quatro auxiliares de enfermagem para o trabalho na maternidade em cada turno de 12 horas. Na unidade neonatal é alocada apenas uma dessas profissionais por turno, com carga horária de trabalho de 36 a 48 horas por semana. Em alguns casos uma incubadora acomoda dois recém-nascidos ao mesmo tempo.

O estudo teve como fonte de informações os livros de registros das salas de parto e da unidade neonatal da referida maternidade, no período de 2007 a 2013. Foram incluídos todos os recém-nascidos de BPN do referido período, com nascimentos ocorridos na maternidade em questão. Participaram do estudo 2,100 bebês de BPN.

A análise dos dados foi realizada com base na estatística descritiva e os resultados estão apresentados em tabelas de frequência absoluta simples e relativa simples, com discussão em consonância com a literatura científica, abordando aspectos sobre limites e benefícios para a promoção do acesso universal à saúde em uma realidade contextualizada.

O projeto foi apreciado e aprovado pelo Comitê de Ética em Pesquisa da Faculdade de Medicina da Universidade Agostinho Neto-Angola, com deliberação no $5 / 14$. Por tratar-se de pesquisa com coleta dos dados em fontes secundárias, foi obtida a dispensa do Termo de Consentimento Livre e Esclarecido.

\section{Resultados}

Nos resultados são apresentados dados maternos e dos bebês com BPN. No período analisado, houve 28,124 nascimentos, sendo 2,100 bebês de BPN, que correspondeu
Table 1. Distribuição da idade materna das gestantes atendidas nas salas de parto e unidades neonatais da Maternidade do Hospital Geral do Bié, segundo peso ao nascer, idade gestacional e consultas no pré-natal; Kuito, Bié, Angola, 2007-2013

\begin{tabular}{|c|c|c|c|c|c|c|}
\hline \multirow[t]{2}{*}{ Idade materna } & \multicolumn{2}{|c|}{$<2,500 \mathrm{~g}$} & \multicolumn{2}{|c|}{$<37$ semanas } & \multicolumn{2}{|c|}{$\begin{array}{l}<6 \text { consultas } \\
\text { no pré-natal }\end{array}$} \\
\hline & $n$ & $\%$ & $n$ & $\%$ & $n$ & $\%$ \\
\hline$<19$ anos & 946 & 45.0 & 53 & 8.0 & 280 & 15.8 \\
\hline 19-34 anos & 964 & 45.9 & 301 & 45.2 & 795 & 44.8 \\
\hline$\geq 35$ anos & 190 & 9.1 & 313 & 46.8 & 697 & 39.3 \\
\hline Total & 2,100 & 100.0 & 667 & 100.0 & 1,772 & 100.0 \\
\hline
\end{tabular}

a 7.5\% dos nascimentos ocorridos na maternidade investigada. Dos 2,100 bebês de BPN, 437 (20.8\%) evoluíram para óbito no primeiro ano de vida, ocorrendo $70.3 \%$ de mortes no período neonatal.

Em relação à procedência, os participantes eram, predominantemente, do município de Kuito-Angola. As gestantes residentes nesse município acorrem preferencialmente a Maternidade do HGB para realizar os partos institucionais pela proximidade dos seus domicílios, mas há também procedentes de outros locais que distam cerca de $180 \mathrm{~km}$. Esses dados, associados às vias rodoviárias em condições inadequadas e aos transportes insuficientes, indicam dificuldades para o acesso ao local do parto e nascimento.

Dos 2,100 bebês com BPN, 349 (16.6\%) nasceram com menos de $1,000 \mathrm{~g}$, denominados de extremo baixo peso ao nascer, $761(36.2 \%)$ entre 1,000 e 1,499 g, de muito baixo peso ao nascer, e 990 (47.2\%) entre 1,500 e $<2,500 \mathrm{~g}$. Todos esses recém-nascidos são vulneráveis e configuram um contexto de alerta e preocupação pela morbimortalidade e prognóstico das condições de saúde e de vida.

A Tabela 1 traz a idade materna em comparação com o BPN, a prematuridade e as consultas no pré-natal.

Observa-se que as mães das faixas etárias $<19$ anos e entre 19 e 34 anos tiveram número expressivo de crianças com BPN. Já nas faixas etárias entre 19 e 34 anos e acima de 35 anos de idade tiveram bebês pré-termos e pouca adesão ao pré-natal.

No estudo, também foram encontrados registros hospitalares referentes às enfermidades presentes na gestação, com ocorrência de malária, HIV/AIDS, hepatites, hipertensão arterial e diabetes entre as gestantes que tiveram bebês com BPN. No tocante ao pré-natal, o número de consultas $<6$ foi de $84.4 \%$ e $\geq 6$ de $15.6 \%$, entre as gestantes com bebês de BPN. Em relação ao tipo de parto, 
Table 2. Distribuição dos óbitos dos BPN atendidos nas salas de parto e unidades neonatais da Maternidade do Hospital Geral do Bié segundo peso ao nascer, idade gestacional, e Apgar no quinto minuto de vida; Kuito, Bié, Angola, 2007-2013

\begin{tabular}{|c|c|c|c|c|c|c|}
\hline \multirow[t]{2}{*}{ Óbitos } & \multicolumn{2}{|c|}{$<2,500 \mathrm{~g}$} & \multicolumn{2}{|c|}{$<37$ semanas } & \multicolumn{2}{|c|}{ Apgar score $<6$} \\
\hline & $n$ & $\%$ & $n$ & $\%$ & $n$ & $\%$ \\
\hline$<7$ dias & 157 & 51.1 & 22 & 15.0 & 64 & 40.3 \\
\hline $7 \mathrm{a}<28$ dias & 150 & 48.9 & 126 & 85.0 & 95 & 59.7 \\
\hline Total & 307 & 100.0 & 148 & 100.0 & 159 & 100.0 \\
\hline
\end{tabular}

Table 3. Distribuição dos BPN atendidos nas salas de parto e unidades neonatais da Maternidade do Hospital Geral do Bié, segundo enfermidades registradas; Kuito, Bié, Angola, 2007-2013

\begin{tabular}{lrc}
\hline Causas óbitos & $n$ & $\%$ \\
\hline Infecção respiratória & 114 & 5.6 \\
Asfixia perinatal e neonatal & 103 & 4.9 \\
Extremamente baixo peso & 55 & 2.6 \\
Síndrome febril & 54 & 2.5 \\
Malária & 53 & 2.5 \\
Síndrome convulsiva & 46 & 2.2 \\
Malformação congénita & 41 & 1.9 \\
Icterícia neonatal & 40 & 1.9 \\
Tétano neonatal & 16 & 0.7 \\
Outras causas & 18 & 0.8 \\
Sem intercorrências & 1,560 & 74.4 \\
\hline Total & 2,100 & 100.0 \\
\hline
\end{tabular}

1,432 (68.2\%) foram partos normais, 563 (26.8\%) cesáreas, e 105 (5.0\%) com uso de ventosa. Cabe salientar que, pela demanda por serviços obstétricos, algumas vezes um leito alberga duas mulheres parturientes, e as puérperas sem complicações têm alta hospitalar em até 12 horas depois do parto.

A Tabela 2 apresenta a comparação entre óbitos neonatais com dados de BPN, a prematuridade e o índice de Apgar no quinto minuto de vida.

Os resultados apontam que no período neonatal precoce ( $<7$ dias de vida) houve mais óbitos de bebês de BPN e no período neonatal tardio (de $7 \mathrm{a}<28$ dias de vida) houve mais óbitos relacionados à prematuridade. $\mathrm{O}$ índice de Apgar no quinto minuto de vida com score menor do que 6 foi expressivo para os óbitos, tanto para os ocorridos no período neonatal precoce quanto no neonatal tardio.

$\mathrm{Na}$ Maternidade estudada, no tocante ao tempo de internação, dos 2,100 bebês com BPN, 800 (38.1\%) tiveram alta nas primeiras 24 horas de vida e desses 103 (12.9\%) foram a óbito no primeiro dia de vida, 761 (36.2\%) ficaram hospitalizados de 1 a $<7$ dias e desses 157 (20.6\%) foram a óbito nos primeiros sete dias de vida, 349 (16.6\%) ficaram hospitalizados de 7 a $<28$ dias e desses 150 (43\%) foram a óbito no período neonatal tardio. Os demais bebês, 190 (9.1\%), ficaram mais do que 28 dias hospitalizados e não houve óbito na internação. Os resultados apontam uma mortalidade ocorrendo precocemente nas unidades neonatais. Cabe destacar que os óbitos extrahospitalares não constam dos registros hospitalares investigados. A Tabela 3 mostra as enfermidades registradas para os bebês de BPN, no período investigado.

$\mathrm{Na}$ Maternidade estudada, os registros apontam dados expressivos de bebês de BPN com ocorrência de infecções respiratórias, asfixia perinatal e neonatal, malária, tétano, extremo baixo peso ao nascer, entre outras. As enfermidades infantis e as da gestação, especialmente as infecciosas, exercem um enorme impacto negativo sobre a saúde materna e infantil para a população estudada. Chama a atenção o elevado percentual de registros "sem intercorrências", podendo denotar insuficiência de informações clínicas no cotidiano da atenção à saúde hospitalar.

Os resultados indicam situações de fragilidade, com danos e repercussões considerados evitáveis e que realçam problemas relacionados ao acesso a recursos e medidas terapêuticas de saúde, impondo limites e desafios para a solução de desigualdades na cobertura das intervenções de saúde e sociais.

\section{Discussão}

O presente estudo aponta expressiva mortalidade neonatal entre os bebês de BPN na realidade estudada, a semelhança de outros cenários africanos $[7,8,15]$. Foram encontradas as seguintes informações maternas e dos recém-nascidos consonantes com condições de vulnerabilidades: idade menor que 19 anos, doenças durante a gestação e gestantes que não participaram satisfatoriamente de consultas no pré-natal, extremo baixo peso ao nascer, muito baixo peso, índice de Apgar menor que 6 e idade gestacional menor que 37 semanas.

As mortes no período neonatal, nos últimos anos, constituem o principal componente da mortalidade infantil em várias regiões do mundo, e esses óbitos são quase todos evitáveis, porém estão se mantendo em níveis elevados, com declínio lento $[1,16]$.

O período neonatal representa o momento mais vulnerável para a sobrevivência de uma criança, sendo cerca 
de $46 \%$ de todas as mortes de menores de cinco anos, em termos mundiais [7].

As condições de vulnerabilidade vinculadas à ocorrência de óbitos neonatais encontradas neste estudo apontam como importantes a idade materna (adolescentes ou com idade igual ou superior a 35 anos), baixa escolaridade materna, problemas relacionados às características reprodutivas, atenção pré-natal, parto e condições de nascimento, presentes na realidade angolana $[8,9]$. Todos esses fatores e as dimensões a eles interrelacionadas podem aumentar as situações de ocorrência da mortalidade neonatal e denotar dificuldades para o acesso à saúde.

$\mathrm{Na}$ África, o período do primeiro dia de nascimento até a segunda semana de vida representa momento crítico para as causas de morte e é nessa ocasião que diminui a cobertura dos cuidados de saúde, com metade das mulheres africanas e dos seus bebês que não se beneficiam dos cuidados dos profissionais de saúde durante o parto e na continuidade da atenção à saúde pós-natal $[7-9,15]$. Na realidade angolana, também já estudada $[15,17]$, a melhoria na qualidade de assistência pré-natal, ao parto e ao recém-nascido de risco é apontada como essencial para reduzir a mortalidade infantil, bem como a melhoria dos condicionantes da elevada mortalidade em crianças, relacionados à falta de saneamento básico, condições inadequadas de abastecimento de água e do acesso aos serviços de saúde. Outro aspeto a ressaltar é a fragilidade da qualidade do sistema de informação em saúde no país, com lacunas mostradas também noutros estudos $[15,17]$.

De acordo com critérios de evitabilidade [18], os resultados do presente estudo apontam que há mortes evitáveis, relacionadas aos grupos reduzíveis por: ações de imunoprevenção, atenção à mulher na gestação, parto e ao recém-nascido, diagnóstico e tratamento adequados e promoção da saúde. O conceito de mortes evitáveis é visto como uma ferramenta útil para o monitoramento do impacto das ações de saúde em relação à probabilidade de mortes, sugerindo que determinados óbitos não deveriam ocorrer, por ser possível sua prevenção e/ou tratamento do agravo ou condições que os determinam [18].

O óbito evitável está relacionado a indicadores sensíveis à qualidade da atenção prestada pelo sistema de saúde, que proporcionam tomadas de decisões. Ainda, pode ser compreendido como evento sentinela, que necessita ser acompanhado, analisado e apresentado como definidor de situações evitáveis e indicador de que a qualidade da atenção deve ser aprimorada, com busca de intervenções sobre possíveis setores socioeconômicos, ambientais, culturais ou genéticos, que possam ser determinantes da situação encontrada [18].

Mortalidade, recém-nascidos de baixo peso ao nascer e acesso universal
A análise de mortes de crianças e mulheres por causas evitáveis, que refletem o funcionamento da rede de atenção à saúde, permite identificar oportunidades perdidas de intervenção pelos serviços de saúde, de modo a reorientar as práticas e evitar novas ocorrências, sendo fundamentais a construção de indicadores, os sistemas de monitoramento da morbimortalidade e a estruturação de comitês de investigação de óbitos infantis e maternos, para avaliação dos serviços e aprimoramento da atenção à saúde $[16,19]$. Portanto, os resultados do presente estudo sugerem a necessidade de melhorar o acesso e a organização dos serviços de saúde, levando em conta uma adequada atenção ao pré-natal, ao parto e ao nascimento.

Em contexto angolano, há recomendações para aumentar a cobertura das intervenções de saúde essenciais, relativas à vacinação, atenção pré-natal e à criança, ações dos Comités de Auditoria de Mortes Maternas e Neonatais a nível provincial, municipal e institucional, medidas legislativas e regulamentares, entre outras, que favoreçam a humanização na atenção à saúde $[8,9,17]$. No entanto, ainda há muitos desafios para incrementar as condições de cuidado e para reduzir vulnerabilidades individuais, sociais e institucionais.

O estudo mostrou, além da importância do baixo peso ao nascer em relação à sobrevivência da criança, a preocupação com a baixa frequência nas consultas de pré-natal e presença de enfermidades na gestação, pela relevância de um pré-natal adequado que reflete melhores resultados perinatais e contribui para diminuir dificuldades e limites da sobrevivência de crianças e mães $[1,16]$. Também, mostra uma preocupação com a ocorrência de cesáreas, em dissonância do que é esperado pelas diretrizes da Organização Munidial da Saúde, que declara que, em nível populacional, taxas de cesárea maiores que $10 \%$ não estão associadas com redução de mortalidade materna e neonatal [20]. Ainda, a prematuridade, os escores baixos de Apgar e as enfermidades registradas das crianças indicam limites das condições assistenciais em saúde e situação de extrema vulnerabilidade $[1,21]$.

O Sistema Nacional de Saúde de Angola enfrenta problemas de cobertura sanitária e para a manutenção das unidades de saúde, com frágil sistema de referência e contra referência entre os níveis de atenção à saúde, recursos humanos e técnicos reduzidos em expressão quantitativa e qualitativa, má distribuição do pessoal nas áreas rurais e urbanas, fragilidades na gestão em saúde, incluindo os sistemas de informação, logística e comunicação, insuficiência de recursos financeiros e inadequação do modelo de financiamento, reduzido acesso à água potável, saneamento e energia $[8,9,17]$. 
Os progressos em busca do incremento do acesso universal à saúde neonatal, infantil e materna têm ficado aquém das expectativas na Região Africana, pois os esforços envidados para melhorar são dificultados por: acesso inadequado e distribuição injusta de serviços de saúde materno-infantil, recursos financeiros inadequados, falta de capacidade institucional e de recursos humanos qualificados, frágil sistema de informação sanitária para acompanhar os progressos realizados, e pouco envolvimento das comunidades [22]. Na realidade angolana, como apontam os resultados do presente estudo, pode ser identificado o preceito do inverse care law, que define que aqueles que têm mais necessidades de cuidado são os que recebem menos cuidados [11]. As crianças e mães pertencentes a grupos socialmente mais vulneráveis recebem, em geral, assistência pré-natal de menor qualidade, com uma oferta desigual de serviços de saúde [19], bem como ocorrem discrepâncias nas questões financeiras do setor saúde, com vistas a assegurar um financiamento sustentável e gestão eficiente dos recursos de saúde [5].

Para promover a justiça social é necessário mensurar e atender a demanda por serviços de saúde das populações mais vulneráveis de um país [5], e a discriminação daqueles que apresentam mais necessidades faz com que os serviços acentuem as diferenças entre os cidadãos ao invés de corrigi-las, sendo necessário mensurar e atender a demanda por serviços de saúde [19]. Em função da necessidade de acelerar a implementação de intervenções de saúde essenciais, são fundamentais as respostas de diretrizes governamentais, da organização e prestação de serviços de saúde, com qualificação de recursos humanos, financeiros e de informação em saúde [22].

Desafios importantes são postos para a solução das mortes evitáveis, em prol do nascimento seguro, redução da alta morbimortalidade infantil e diminuição da alta frequência de nascimentos pré-termo e de baixo peso ao nascer [1]. As causas de mortalidade neonatal precoce são possíveis de prevenção ao alinharem condutas adequadas durante o curso da gestação, no momento do parto e nascimento e na atenção longitudinal em saúde e, desse modo, repercutirem em melhoria do acesso universal à saúde $[6,21]$.

$\mathrm{O}$ acesso às intervenções de saúde dirigidas às crianças e mulheres necessita ser substancialmente ampliado, em busca de coberturas universais e superação de desigualdades de acesso. Iniciativas para monitorar a qualidade dos resultados em saúde são de extrema relevância para assegurar que crianças em situação de vulnerabilidade recebam um cuidado com custo efetivo e equitativo [23, 24], para lidar com efeitos da privação e das iniquidades
[11]. Assim, tem-se como desafios a necessidade de melhoria nos determinantes sociais (pobreza, educação, urbanização e fecundidade) [21], nos sistemas de registro e notificação de óbitos e ampliação das investigações de óbitos de crianças e de mulheres [16], incremento da atenção primária à saúde e articulação entre os setores sociais para além do âmbito da saúde $[3,21]$, com capacidade de responder de acordo com as necessidades das pessoas e populações.

Para avançar nas questões que envolvem o acesso à saúde é fundamental a análise contínua das diversas barreiras e condições de cuidado em saúde, com forte ampliação do número de profissionais de saúde qualificados [25], particularmente no campo da atenção primária à saúde, para promoção ampla de acesso com cuidados de saúde primários de qualidade.

As limitações do presente estudo referem-se aos sistemas insuficientes de informação em saúde, com lacunas e fragilidades no armazenamento e conservação formal do arquivo clínico na instituição estudada.

\section{Conclusão}

No presente estudo foi possível analisar aspectos da mortalidade neonatal de bebês de BPN, relevantes para auxiliar no planejamento de ações para a reestruturação e melhoria da atenção à saúde de recém-nascidos e gestantes, visando à redução de mortes evitáveis na realidade angolana.

A diminuição dos óbitos neonatais não depende fundamentalmente de novos conhecimentos, mas da garantia do acesso universal à saúde e da utilização mais efetiva do conhecimento científico e tecnológico existente. Os critérios de evitabilidade de óbitos em crianças incluem uma ampla análise das condições sociais e dos serviços de saúde, levando-se em conta uma adequada atenção ao pré-natal, ao parto, ao nascimento e no seguimento longitudinal, em busca do acesso à saúde com equidade, intervenções profissionais qualificadas e gestão da saúde.

Assim, no contexto identificado, a melhoria do acesso à saúde e as intervenções para a redução de vulnerabilidades sociais, institucionais e individuais podem conferir relevantes modificações rumo ao cuidado integral à saúde, defesa de direitos humanos e cidadania.

\section{Agradecimentos}

Ao Conselho Nacional de Desenvolvimento Científico e Tecnológico - CNPq - Processo no 309085/2015-2. 


\section{References}

1 Liu L, Oza S, Hogan D, Perin J, Rudan I, Lawn JE, et al. Global, regional, and national causes of child mortality in 2000-13, with projections to inform post-2015 priorities: an updated systematic analysis. Lancet. 2015 Jan; 385(9966):430-40.

2 Organización Mundial de la Salud. Monitoreo del progreso hacia la cobertura universal de salud a nivel nacional y global: marco de trabajo, medidas y metas. [Internet]. Geneva: OMS. Banco Mundial; 2014. [Accessed Jan 6, 2016]. Available from: http://apps.who.int/ iris/bitstream/10665/112827/1/WHO_HIS_ HIA_14.1_spa.pdf

3 Sachs J. The MDG decade: looking back and conditional optimism for 2015. Lancet. 2010 Sep;376(9745):950-1.

4 Hecksher M. Um balanço social do milênio. Rev Desafios Desenvolvimento. 2014;11:6470.

5 Noronha JC. Universal health coverage: how to mix concepts, confuse objectives, and abandon principles. Cad Saude Publica. 2013 May;29(5):847-9.

6 Rodin J, de Ferranti D. Universal health coverage: the third global health transition? Lancet. 2012 Sep;380(9845):861-2.

7 World Health Organization. Global Health Observatory (GHO) data: Neonatal mortality: situation and trends. [Internet]. Geneva: World Health Organization; 2016. [Accessed Jan 6, 2016]. Available from: http://www.who. int/gho/child_health/mortality/neonatal_ text/en/

8 Organização Mundial de Saúde. Escritório Regional Africano. Estratégia de cooperação da OMS 2015-2019. [Internet]. Luanda, Angola: Organização Mundial de Saúde. Escritório Regional Africano; 2016. [Accessed Jan 6, 2016]. Available from: http://www.who. int/iris/handle/10665/250516

9 Angola. Instituto Nacional de Estatística. Inquérito de indicadores múltiplos e de saúde em Angola 2015-2016. [Internet]. Luanda, Angola: Instituto Nacional de Estatística (INE). Ministério da Saúde (MINSA). Minis- tério do Planeamento e do Desenvolvimento Territorial (MINPLAN). ICF; 2017. [Consulted Jan 2016]. Available from: https://dhsprogram.com/pubs/pdf/FR327/FR327.pdf

10 Britto PR, Ulkuer N. Child development in developing countries: child rights and policy implications. Child Dev. 2012 Jan-Feb;83(1): 92-103.

11 Rahman FR, Maharaj V, Yates R, Beeley C, Moore I, Rose A, et al. Addressing the inverse care law: the role of community paediatric services. Perspect Public Health. 2014 Mar; 134(2):85-92.

12 Barros AJ, Ronsmans C, Axelson H, Loaiza E, Bertoldi AD, França GV, et al. Equity in maternal, newborn, and child health interventions in Countdown to 2015: a retrospective review of survey data from 54 countries. Lancet. 2012 Mar;379(9822):1225-33.

13 Werneck G. Epidemiologia descritiva: qualidade das informações e pesquisa nos serviços de saúde. Epidemiol Serv Saude. 2009;18: 205-7.

14 Organização Mundial da Saúde. Angola: Estratégia de cooperação da OMS com os países 2009-2013: [Internet]. Luanda, Angola: OMS; 2010. Available from: http://www.afro.who. int/pt/angola/representacao-da-oms-angola. html

15 Grady SC, Frake AN, Zhang Q, Bene M, Jordan DR, Vertalka J, et al. Neonatal mortality in East Africa and West Africa: a geographic analysis of district-level demographic and health survey data. Geospat Health. 2017 May;12(1):501.

16 Kassar SB, Melo AM, Coutinho SB, Lima MC, Lira PI. Determinants of neonatal death with emphasis on health care during pregnancy, childbirth and reproductive history. J Pediatr (Rio J). 2013 May-Jun;89(3):269-77.

17 Simão R, Gallo PR. Mortes infantis em Cabinda, Angola: desafio para as políticas públicas de saúde. Rev Bras Epidemiol. 2013;16: 826-37.

18 Malta DC, Sardinha LM, Moura L, Lansky S, Leal MC, Szwarcwald CL, et al. Update of avoidable causes of deaths due to interventions at the Brazilian Health System. Epidemiol Serv Saude. 2010;19:173-6.

19 Lima MC, de Oliveira GS, Lyra CO, Roncalli AG, Ferreira MA. [The spatial inequality of low birth weight in Brazil]. Cien Saude Colet. 2013 Aug;18(8):2443-52.

20 Organização Mundial da Saúde. Declaração da OMS sobre taxas de cesáreas. [Internet]. Genebra, Suíça: Departamento de Saúde Reprodutiva e Pesquisa. OMS; 2014. [Accessed Jan 6, 2016]. Available from: http://apps.who. int/iris/bitstream/10665/161442/3/WHO_ RHR_15.02_por.pdf?ua $=1$

21 Victora CG, Aquino EM, do Carmo Leal M, Monteiro CA, Barros FC, Szwarcwald CL. Maternal and child health in Brazil: progress and challenges. Lancet. 2011 May;377(9780): 1863-76.

22 Organização Mundial Saúde. Acelerar o acesso universal à saúde sexual e reprodutiva: agenda para a região africana da Organização Mundial da Saúde. [Internet]. Brazzaville, República do Congo: OMS; 2013. [Accessed Jan 6, 2016]. Available from: https://afro.who. int/sites/default/files/2017-06/RWH-Accelerating-Universal-Access-to-Sexuel-Reproductive-Health-en.pdf

23 Szilagyi PG. Health insurance and children with disabilities. Future Child. 2012;22(1): $123-48$

24 Maharaj V, Rahman F, Adamson L. Tackling child health inequalities due to deprivation: using health equity audit to improve and monitor access to a community paediatric service. Child Care Health Dev. 2014 Mar; 40(2):223-30.

25 Pan American Health Organization. Resolution CD52.R13. Human resources for health: increasing access to qualified health workers in primary health care-based health systems. In: 52nd Directing Council: 65th Session of the Regional Committee. [Internet]. Washington, DC: Pan American Health Organization; 2013. [Accessed Jan 6, 2016]. Available from: http:// iris.paho.org/xmlui/handle/123456789/4441
Mortalidade, recém-nascidos de baixo peso ao nascer e acesso universal
Port J Public Health 2018;36:95-101 DOI: $10.1159 / 000493959$ 